\title{
ESTUDO ARQUEOMETALÚRGICO DO CONJUNTO METÁlICO DO SÍTIO ARQUEOLÓGICO DE MOINHOS DE GOLAS (MONTALEGRE, NORTE DE PORTUGAL): PRIMEIROS RESULTADOS
}

\author{
J. LOUREIRO ${ }^{(1)}$, E. FIGUEIREDO ${ }^{(2,3)}$, R.J.C. SILVA ${ }^{(3)}$, M.F. ARAÚJO ${ }^{(2)}$, J. FONTE ${ }^{(4)}$ \& A.M.S. BETTENCOURT ${ }^{(5)}$
}

Resumo:

Abstract:

\begin{abstract}
O presente trabalho apresenta os primeiros resultados de um estudo arqueometalúrgico detalhado, ainda em curso, efetuado sobre um conjunto de achados metálicos encontrado no sítio de Moinhos de Golas, freguesia de Solveira, concelho de Montalegre. Este, genericamente, atribuído à Proto-história foi já objeto de uma primeira publicação (FONTE et al. 2013)

A coleção é composta por 35 objetos, entre os quais se encontram armas, artefactos de adorno e utensílios, entre outros de difícil classificação funcional.

Foram efetuadas radiografias por raios $\mathrm{X}$, análises elementares por micro-EDXRF e observações microestruturais por microscopia ótica na maioria dos artefactos. As imagens radiográficas revelaram algumas heterogeneidades estruturais nalgumas peças e as análises elementares demonstraram que a maioria dos artefactos foi produzida numa liga de bronze binária $(\mathrm{Cu}-\mathrm{Sn})$, sendo, no entanto, de destacar o uso de outros metais e ligas, como, por exemplo, cobre e latão. Foram, ainda, observadas microestruturas associadas a diferentes processos de manufatura que poderão envolver ciclos de martelagem e recozimento, com ou sem operações de acabamento.
\end{abstract}

Palavras-chave: Arqueometalurgia, Bronze, Composição elementar, Técnicas de manufatura, Técnicas analíticas

\begin{abstract}
First results of an archaeometallurgical study of a collection from Moinhos de Golas archaeological site (Montalegre, Portugal)

The present work presents the first results of a detailed archaeometallurgical study, still ongoing, on a group of metallic findings from Moinhos de Golas archaeological site (Montalegre, Portugal), generically attributed to the Protohistory, and previously presented in a paper dealing with its discovery (FONTE et al. 2013).

The collection is composed by 35 objects, which include weapons, ornaments and tools, among other objects of a more difficult classification.

The archaeometallurgical study involved X-ray radiography, elemental analysis by micro-EDXRF and microstructural observations by optical microscopy. The radiographies revealed some structural heterogeneities in some artefacts and the elemental analysis showed that most of the artefacts were produced in a binary bronze alloy $(\mathrm{Cu}-\mathrm{Sn})$, being others made in copper and brass. Microstructures associated with different manufacturing processes were observed, which may involve hammering and annealing cycles with or without finishing operations.
\end{abstract}

Keywords: Archaeometallurgy, Bronze, Alloy composition, Manufacturing techniques, Analytical techniques

Received: 31 July, 2014; Accepted: 23November, 2014

\section{INTRODUÇÃO}

Os achados arqueológicos metálicos possuem um carácter único e fazem parte de uma herança cultural com um elevado valor histórico e tecnológico. Desta forma, é importante o seu estudo e a sua preservação com o mínimo de alteração possível, não só ao nível da aparência, como ao nível das suas propriedades físicas e químicas (LEHMANN et al. 2005). Neste sentido, são relevantes os projetos multidisciplinares, que abordem várias áreas de estudo em torno dos artefactos e coleções, e que incluam a aplicação de técnicas de exame e análise adaptadas à natureza de cada objeto cultural em particular. A contribuição dos estudos analíticos, através duma aproximação multi-analítica, torna-se relevante para a identificação dos materiais e processos de manufatura, contribuindo para um melhor entendimento da história e tecnologia das sociedades passadas, bem como a utilização de materiais primitivos e a sua degradação (ADRIENS

\footnotetext{
(1) Departamento de Conservação e Restauro, Faculdade de Ciências e Tecnologia, Universidade Nova de Lisboa, 2829-516 Monte de Caparica, Portugal. jg.loureiro@campus.fct.unl.pt

${ }^{(2)}$ Centro de Ciências e Tecnologias Nucleares $\left(C^{2} \mathrm{TN}\right)$, Instituto Superior Técnico, Universidade de Lisboa, Estrada Nacional 10, ao km 139,7, 2695-066 Bobadela LRS, Portugal. elin@ctn.ist.utl.pt (Elin Figueiredo); faraujo@ctn.ist.utl.pt (M.Fátima Araújo).

(3) Centro de Investigação de Materiais (CENIMAT/I3N), Departamento de Ciências dos Materiais, Faculdade de Ciências e Tecnologia, Universidade Nova de Lisboa, 2829-516 Monte de Caparica, Portugal. emf12055@campus.fct.unl.pt (Elin Figueiredo); rjcs@fct.unl.pt (Rui J.C. Silva)

(4) Instituto de Ciencias del Patrimonio (Incipit), Consejo Superior de Investigaciones Cientificas (CSIC), 15782 Santiago de Compostela, Espanha. joao.fonte@incipit.csic.es

${ }^{(5)}$ Centro de Investigação Transdisciplinar "Cultura, Espaço e Memória" (CITCEM/UM), Departamento de História, Universidade do Minho, Campus de Gualtar, 4710-057 Braga, Portugal. anabett@uaum.uminho.pt
} 


\section{4; WAYMAN 2000)}

No presente trabalho apresentam-se os primeiros resultados de um estudo arqueometalúrgico detalhado da coleção metálica de Moinhos de Golas (Solveira, Montalegre, Vila Real), cujo contexto e condições de achado foram dados a conhecer recentemente (FONTE et al. 2013). De destacar que no sítio arqueológico foram encontrados, além dos materiais metálicos, várias dezenas de fragmentos de panças cerâmicas, todas elas de fabrico manual, pastas arenosas, cozeduras redutoras, de acabamento alisado e sem decoração, características similares às restantes produções da Idade do Bronze do Noroeste Peninsular que, nesta região, se podem prolongar até aos séculos VII/VI a.C.. De notar, ainda, que nas várias plataformas onde ocorrem os artefactos metálicos, o acervo cerâmico não apresenta diferenças.

A coleção metálica é composta por 35 objetos, entre os quais se encontram: armas (três punhais de diferentes formas e dimensões); artefactos de adorno (um botão e dois alfinetes); utensílios (um tranchet, um eventual freio e dois potenciais passadores de correia); entre outros de difícil classificação funcional (como argolas, um cravo, uma placa fragmentada, varetas, hastes e um objeto em forma de cápsula) (FONTE et al. 2013). Os punhais e o tranchet apresentam tipologias típicas do Bronze Final, sendo que as argolas, os rebites ou cravos, as varetas e os botões são conhecidos em contextos do Bronze Final do Centro e Noroeste da Ibéria (SENNA-MARTINEZ 1989; CARDOSO 1995; VILAÇA 1995; BETTENCOURT 1998; BOTTAINI 2012) sendo que algumas peças persistem nos inícios da Idade do Ferro do Noroeste. Os restantes objetos poderão também ser de cronologias posteriores, dado o seu contexto de achado.

Assim, neste estudo arqueometalúrgico irão ser discutidos os resultados obtidos através de uma combinação de técnicas de exame e análise que fornecem informações complementares sobre os artefactos, nomeadamente a nível composicional, macro e micro estrutural, incluindo: a radiografia por raios $\mathrm{X}$, a espectrometria de fluorescência de raios $\mathrm{X}$ (micro-EDXRF) e a microscopia ótica.

A radiografia por raios $X$ é um método de exame, não destrutivo, que possibilita o estudo da estrutura interna dos artefactos respeitando a integridade física do material/objeto, não sendo este alterado pelo procedimento analítico (ADRIENS 2005; FIGUEIREDO et al. 2011). No presente trabalho foi usado para auxiliar o estudo das técnicas de manufatura e avaliar o estado de conservação dos diversos artefactos.

A espectrometria de fluorescência de raios $X$ (XRF) é uma técnica analítica multielementar, relativamente rápida e fácil, adequada para identificar e quantificar a maioria dos constituintes das ligas primitivas (VALÉRIO et al. 2007). Permite estudar a composição elementar de forma não destrutiva, na medida em que as análises são feitas sobre a superfície a estudar sem a alteração química ou física desta.
Contudo, devido ao facto de os artefactos arqueológicos terem permanecido enterrados no solo por longos períodos de tempo, possuem uma camada superficial alterada (camada de corrosão) cuja composição é diferente da liga original, podendo estar enriquecida em determinados elementos de liga metálica (p. ex., em ligas de bronze, a camada superficial corroída sofre lixiviação preferencial do cobre (RoBBIOLA et al. 1998) resultando em teores de estanho superiores quando comparado com a liga original). Adicionalmente, a superfície poderá encontrar-se contaminada com elementos do ambiente de deposição como, por exemplo, o ferro presente no solo. Assim, torna-se necessária a remoção das camadas superficiais de corrosão, de forma a se poder analisar o metal inalterado. Para o presente estudo foi utilizada a micro espectrometria de fluorescência de raios $\mathrm{X}$, dispersiva de energias (micro-EDXRF), que permite a realização de análises numa pequena área da superfície livre das camadas de corrosão.

A observação microestrutural por microscopia ótica permite obter informações relevantes para o entendimento dos processos e técnicas de manufatura das diferentes tipologias de objetos através da visualização da microestrutura de cada artefacto em particular. É também uma técnica importante para o estudo e identificação de processos de degradação que poderão, por sua vez, ter implicações na preservação futura das coleções. No presente trabalho, e tendo em conta as características específicas do equipamento de microscopia ótica disponível (vide detalhes na seção do procedimento experimental e em FIGUEIREDO et al. 2013b), a observação das microestruturas foi efetuada na pequena área limpa das camadas de corrosão superficiais, onde também foram efetuadas as análises de microEDXRF, não sendo, portanto, necessária a amostragem.

\section{PROCEDIMENTO EXPERIMENTAL}

A radiografia por raios $X$ foi conduzida num sistema de radiografia digital, ArtXRay SEZ Series, fabricado pela NTB GmbH (Dickel, Alemanha). Foi utilizada uma diferença de potencial de $130 \mathrm{kV}$, uma intensidade de corrente de 3,7 mA, correspondente a uma potência de 480 W. A geometria de análise incluiu uma distância da ampola ao detetor de $1,36 \mathrm{~m}$, com os artefactos posicionados a cerca de $14 \mathrm{~cm}$ do detetor. O processamento de imagem envolveu o software iXPect.

Para as análises elementares por micro-XRF e observação microestrutural por microscopia ótica, os objetos foram sujeitos a um desbaste superficial de uma pequena área $\left(<5 \mathrm{~mm}^{2}\right)$, para retirar as camadas de corrosão superficiais e a um posterior polimento manual, com pastas de diamante de diferentes granulometrias (de 6 a 1 
$\mu \mathrm{m})$ em cotonetes, de forma a obter uma superfície metálica limpa e adequada para o estudo da liga inalterada.

As análises de micro-EDXRF foram efetuadas num espectrómetro ArtTAX Pro (Fig. 1), equipado com um tubo de raios $\mathrm{X}$ de baixa energia com um ânodo de Mo, um detetor de silício eletroquimicamente refrigerado com uma resolução de $160 \mathrm{eV}(\mathrm{Mn}-\mathrm{K} \alpha)$, um conjunto de lentes policapilares que geram um feixe de radiação primária que irá incidir numa micro área de $\sim 70 \mu \mathrm{m}$ de diâmetro e uma câmara CCD integrada (BRONK et al. 2001). As condições selecionadas para as presentes análises foram 40 $\mathrm{kV}$ de diferença de potencial na ampola, $600 \mu \mathrm{A}$ de intensidade de corrente e $100 \mathrm{~s}$ de tempo de aquisição. A quantificação dos artefactos envolveu a utilização do software Winaxil e uma calibração utilizando um material certificado de referência (Phosphor Bronze 551 da British Chemical Standards (BCS)). Detalhes acerca do procedimento de quantificação foram descritos recentemente (FIGUEIREDO et al. 2013a).

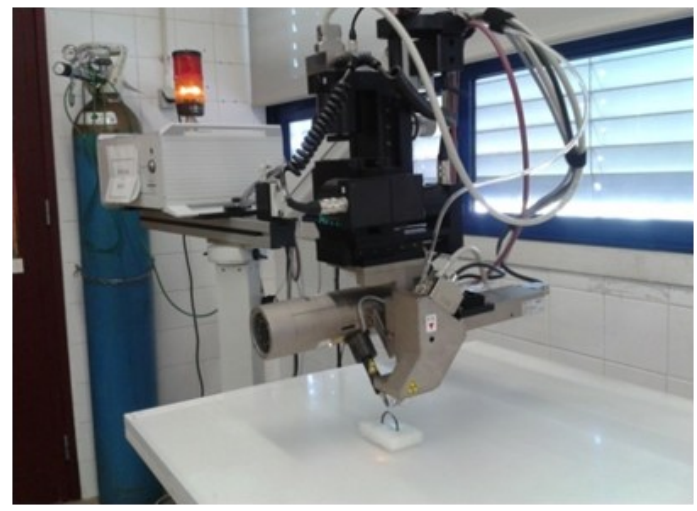

Fig. 1. Análise de uma argola por micro-EDXRF no espectrómetro ArtTAX Pro instalado no Departamento de Conservação e Restauro da FCT-UNL.

Fig. 1. Micro-EDXRF analysis of a ring by the ArtTAX spectrometer installed at the Department of Conservation and Restoration in FCT-UNL.

As observações microestruturais foram efetuadas num microscópio de reflexão Leica DMI5000M de objetivas invertidas, em campo claro e sob luz polarizada, e registadas com uma câmara fotográfica digital acoplada (Fig. 2). As características particulares do microscópio possibilitam o estudo de artefactos sem implicar a recolha prévia de amostras. Adicionalmente, a automatização do equipamento controlada pelo software Leica Application Suite permite recorrer à funcionalidade multifocus, essencial para a observação de superfícies menos planas, como as resultantes de uma preparação metalográfica manual e localizada (FIGUEIREDO et al. 2013b).

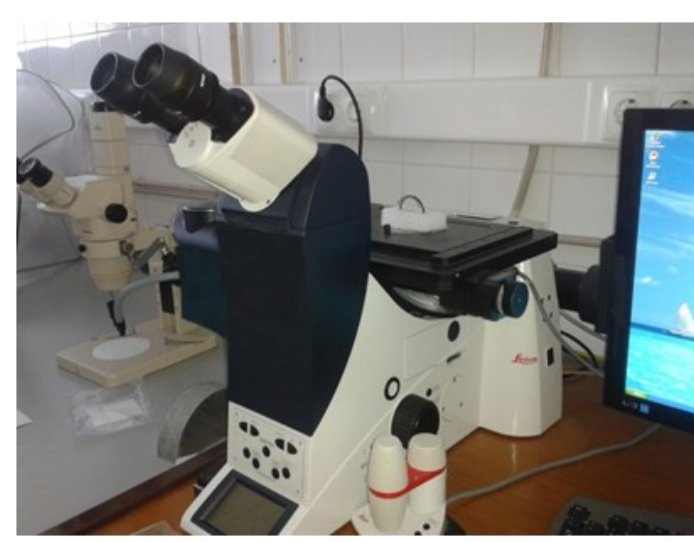

Fig. 2. Observação de uma argola por Microscopia Ótica no microscópio Leica DMI5000M instalado no CENIMAT/ I3N da FCT-UNL.

Fig. 2. Examination of a ring by Optical Microscopy on a Leica DMI5000M optical microscope installed at CENIMAT/I3N in FCT-UNL.

\section{PROCEDIMENTO EXPERIMENTAL}

\subsection{Radiografia digital por raios $X$ - Estrutura interna dos artefactos e avaliação do estado de conservação}

As imagens obtidas por radiografia de raios $\mathrm{X}$ foram processadas digitalmente de forma a que as áreas mais escuras correspondessem a densidades e/ou espessuras mais elevadas e as áreas mais claras a densidades e/ou espessuras mais baixas.

As radiografias dos alfinetes 2013-0448 e 2013-0449 apresentam duas imagens opacas (Fig. $3 \mathrm{~A}$ e 3B) sugerindo que são dois artefactos maciços e não ocos. Embora não seja possível observar qualquer vestígio da união entre a haste e a cabeça dos alfinetes, as análises por micro-EDXRF feitas nestes elementos mostram uma composição bastante semelhante, o que poderá sugerir que estes dois artefactos foram fundidos como uma peça só.

Nas radiografias efetuadas ao cravo em diferentes posições também não é possível observar qualquer heterogeneidade que sugira a união de duas peças distintas, isto é, a ligação entre a haste e o cabo (Fig. 3C), o que poderá indicar que este artefacto foi fundido como uma única peça, sendo depois possivelmente trabalhado termomecanicamente.

Nalguns artefactos foram identificadas zonas fragilizadas, com a presença de fissuras e/ou fraturas. Exemplo disso é a presença de uma fissura na zona de maior curvatura de uma pequena vareta (2013-0455) (Fig. 3D), que poderá estar relacionada com tensões internas provocadas pelos processos de manufatura, nomeadamente pela deformação necessária à sua dobragem. No tranchet (2013-0426) (Fig. 3E) também se observaram heterogeneidades na sua estrutura interna: uma zona mais escura, mais densa, à esquerda (assinalada com uma seta na figura) e uma outra 
mais heterogénea, à direita e abrangendo a zona inferior do gume, aparentando ter várias zonas com corrosão localizada. Estas diferenças poderão relacionar-se com distintas intensidades de corrosão, como pode ser depreendido da observação da existência de uma camada de corrosão superficial, mais espessa e possivelmente de carácter mais protetor, na zona esquerda e superior do artefacto, correspondente à zona assinalada com a seta na radiografia (Fig. 3E) visível na fotografia do artefacto publicada em Fonte et al.
(2013: Fig. 6). Os estudos microestruturais efectuados a este artefacto mostram que a zona mais densa, assinalada com uma seta na radiografia, apresenta uma microestrutura de grão mais grosseiro do que a zona mais inferior da lâmina, que exibe uma microestrutura de grão recristalizado com bandas de deformação. Esta característica mostra que a região assinalada na radiografia terá sido menos trabalhada do que a zona inferior da lâmina.

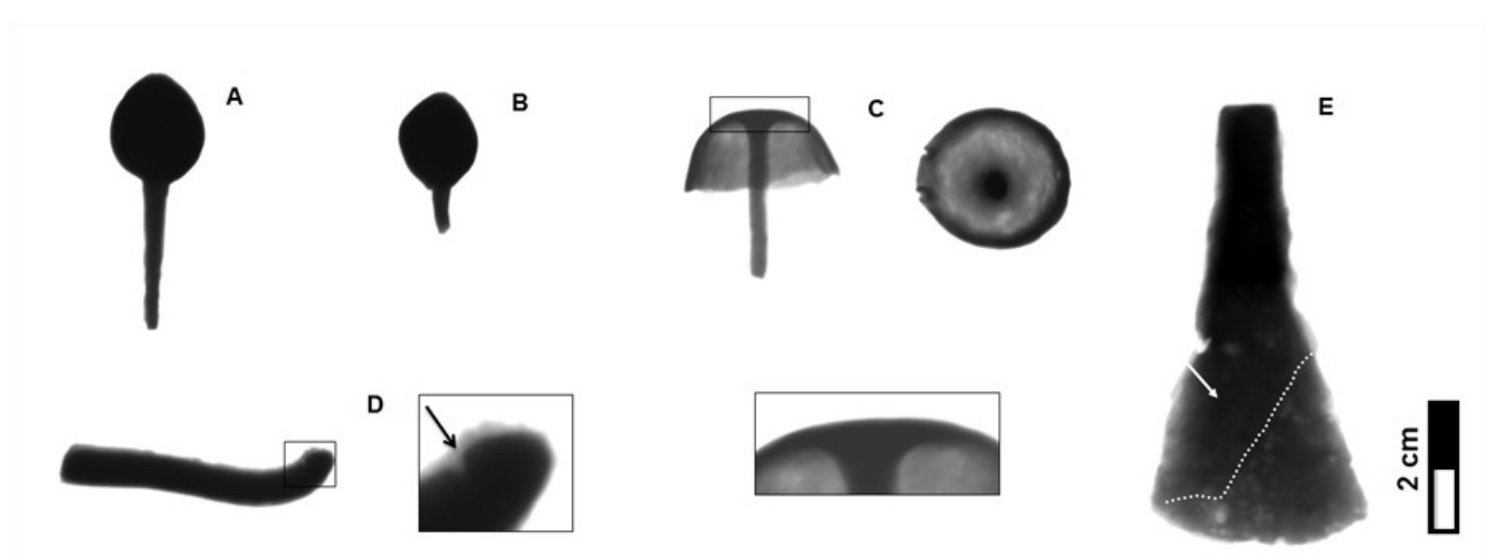

Fig. 3. Radiografia por raios X dos alfinetes 2013-0448 (A) e 2013-0449 (B), do cravo 2013-0447 (C), da vareta 2013-0455 (D), e do tranchet 2013-0426 (E).

Fig. 3. X-ray radiography of the pins 2013-0448 (A) and 2013-0449 (B), the nail 2013-0447 (C), the bar 2013-0455 (D) and the tranchet 2013-0426 (E).

\subsection{Análises por micro-EDXRF - Composição elmentar dos artefactos}

As análises por micro-EDXRF efetuadas na coleção mostram que a maioria dos artefactos é composta por uma liga de bronze binário (i.e., uma liga de $\mathrm{Cu}-\mathrm{Sn}$ ), havendo seis objetos em cobre (três argolas, um elemento de possível freio, uma vareta e uma placa) e três em latão (liga $\mathrm{Cu}-\mathrm{Zn}$ ) (um objeto em forma de cápsula e duas argolas) (Fig. 4).

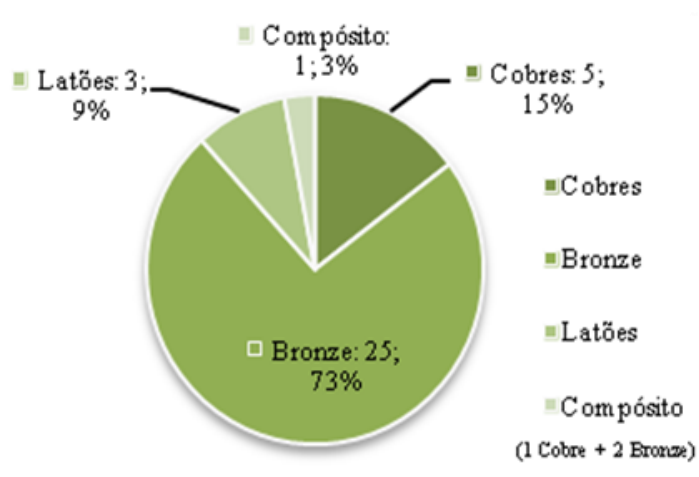

Fig. 4. Distribuição dos artefactos por tipos de metal/ligas metálicas.

Fig. 4. Artefacts distribution by metal/alloy type.
Os objetos em latão não serão discutidos neste trabalho pois, dada a habitual ausência deste tipo de ligas em coleções de artefactos proto-históricos, a existência de tais artefactos terá necessariamente de ser analisada de uma forma mais abrangente através de um estudo mais individualizado.

Tanto nos objetos de bronze como nos de cobre é frequente a presença de $\mathrm{As}, \mathrm{Pb}$ e/ou $\mathrm{Ni}$ em percentagens bastante baixas, sendo interpretada como impurezas associadas às matérias primas.

Relativamente aos bronzes, que representam o maior conjunto dos artefactos, os teores de estanho situam-se maioritariamente entre os 10 e os $15 \%$ (as exceções são uma vareta com $\sim 5 \% \mathrm{Sn}$ e uma argola com $\sim 8 \%$ Sn) (Fig. 5).

Esta composição de liga, juntamente com o baixo teor em impurezas, encontra paralelos nas tradições metalúrgicas do Bronze Final para o território nacional. (cf. diversos autores citados em FIGUEIREDO et al. 2011).

Este intervalo na composição em estanho confere propriedades mecânicas e de coloração particulares ao metal. Um bronze com teores $>10 \%$ $\mathrm{Sn}$ pode ter a sua fase $\mathrm{Cu}$-alfa substancialmente endurecida por solução sólida de $\mathrm{Sn}$, tornando-se muito mais duro do que o cobre (LECHTMAN 1996). Esta elevada dureza, associada a alguma perda de ductilidade, pode ser recuperada através de um recozimento, pelo menos em objetos com teores 
até $15 \% \mathrm{Sn}$, pois é este o limite de solubilidade de $\mathrm{Sn}$ na fase $\mathrm{Cu}$-alfa, sendo por isso o teor mínimo em Sn para a formação de uma fase mais dura, a fase $\delta$, rica em estanho (detalhes no diagrama de fases para o bronze em condições de recozimento, em CENTRE TEChNIQUe DES INDUSTRIES DES Alliages CUIVREUX 1967). Adicionalmente, dentro deste intervalo de composições de ligas de bronze é obtida a coloração mais próxima do ouro (FANG \& MCDONNELL 2011), sendo possível que esta tonalidade dourada pudesse ser significante na época.

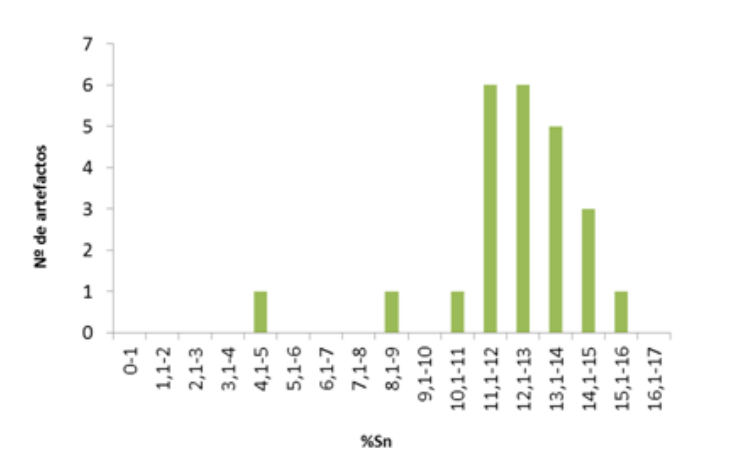

Fig. 5. Histograma de dispersão dos teores de Sn nos artefactos de bronze.

Fig. 5. Histogram of tin content in the bronze artefacts.

De destacar, na coleção, a coexistência de diferentes metais dentro de uma mesma tipologia (como as argolas) e, particularmente, num mesmo artefacto, o eventual freio (2013-0428), que revelou ser um objeto composto, com o arco produzido em cobre (Fig. 6) e as duas argolas

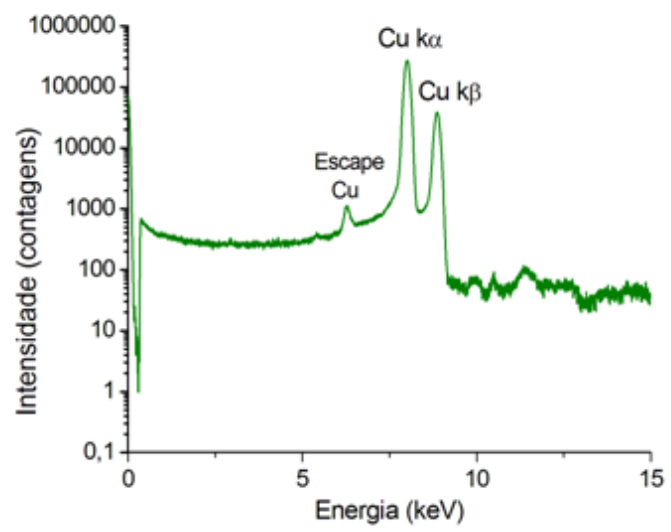

Fig. 6. Espectro de micro-EDXRF referente ao arco em cobre do eventual freio (2013-0428).

Fig. 6. Micro-EDXRF spectrum of the copper rim in the possible horse brake (2013-0428)

em bronze (Fig. 7). A escolha dos diferentes materiais neste artefacto poderá estar relacionada com as propriedades mecânicas dos mesmos, isto é, o cobre terá sido usado por se tratar de um material mais maleável do que o bronze, sendo mais fácil a obtenção da forma fina e em arco, ou por questões estéticas, atendendo a que o cobre tem um tom mais avermelhado, face ao tom dourado do bronze, constituinte das argolas, resultando numa peça com variações cromáticas.

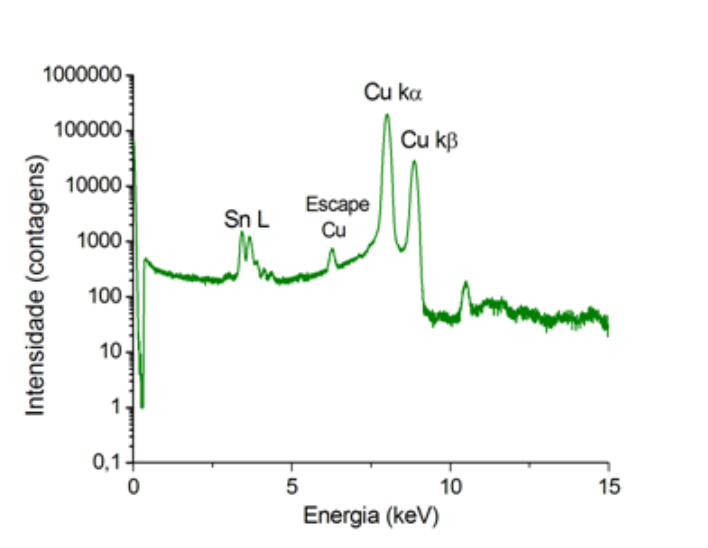

Fig. 7. Espectro referente a uma das argolas em bronze do eventual freio (2013-0428).

Fig. 7. Micro-EDXRF spectrum of a bronze ring in the possible horse brake (2013-0428).

Além do arco do eventual freio, os restantes artefactos em cobre são duas argolas, uma vareta (2013-0453) e a placa dobrada (2013-0456). A manufatura destes artefactos poderá estar, também, relacionada com as propriedades particulares deste material, nomeadamente a coloração distinta do bronze, ou a sua maleabilidade, que poderia ser particularmente relevante para a obtenção da espessura desejada na placa.

A diversidade material observada, i.e., a presença de bronzes, cobres e latões, bem como a diversidade tipológica dos artefactos da coleção poderá apontar para a presença de vários momentos de deposição (não sendo de excluir atos acidentais) e/ou à circulação de materiais exógenos durante a Protohistória. No entanto, é de destacar que as argolas, embora construídas com diferentes materiais metálicos, se assemelham entre si, o que pode sugerir o uso de processos de reciclagem na Proto-história.

\subsection{Microscopia Ótica - Avaliação da corrosão e das técnicas de manufatura}

A observação das superfícies polidas dos artefactos por microscopia ótica e através de luz polarizada permitiu a visualização de diversas camadas de corrosão.

De uma forma geral, a maioria dos artefactos observados revelou uma camada exterior, de tom esverdeado, e uma camada interior de tom mais avermelhado (Fig. 8), que em vários artefactos se estende para o interior dos artefactos ao longo dos limites de grão do metal (corrosão intergranular), uma característica comum em artefactos arqueológicos e resultante de uma corrosão de longo termo. Estas colorações distintas estarão relacionadas com 
diferentes estados de oxidação do cobre, sendo que a região exterior corresponderá a um estado de oxidação mais elevado, o $\mathrm{Cu}[\mathrm{II}]$, frequentemente associado à presença de carbonatos básicos de cobre. Na camada mais interior o estado de oxidação do cobre será menor, $\mathrm{Cu}[\mathrm{I}]$, e a sua coloração avermelhada estará associada à cuprite, $\mathrm{Cu}_{2} \mathrm{O}$ (PICCARDO et al. 2007).

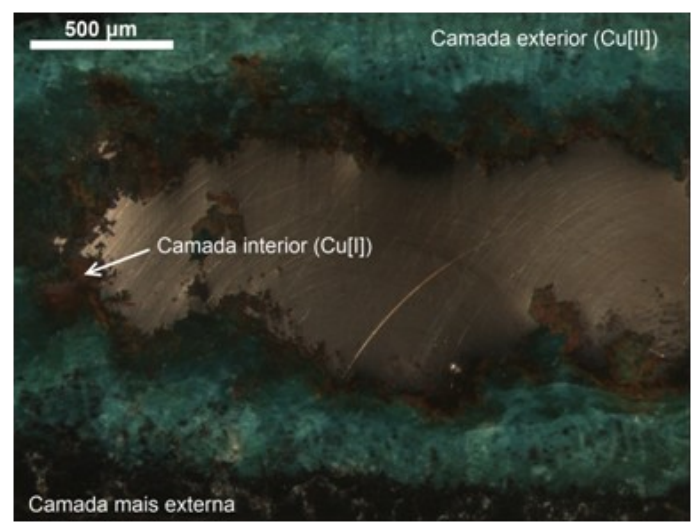

Fig. 8. Observação por microscopia ótica, sob luz polarizada, da zona polida da argola 2013-0436 com visualização das diferentes camadas de corrosão envolvendo o metal inalterado (no centro) $\left(50^{\prime}\right)$.

Fig. 8. Visualization by optical microscopy, under polarized light, of different corrosion layers in the polished surface of the ring 2013-0436 (50').

Em alguns objetos foi possível visualizar uma camada mais externa de tonalidade castanha escura (Fig. 8) que poderá ser entendida como um agregado de produtos de corrosão incorporando partículas do solo provenientes do local de enterramento.

Foram visualizados casos em que a camada mais interior, a avermelhada, se estendeu para o interior do artefacto (Fig. 9) ao longo das fronteiras de grão, revelando consequentemente os tamanhos e as formas dos grãos.

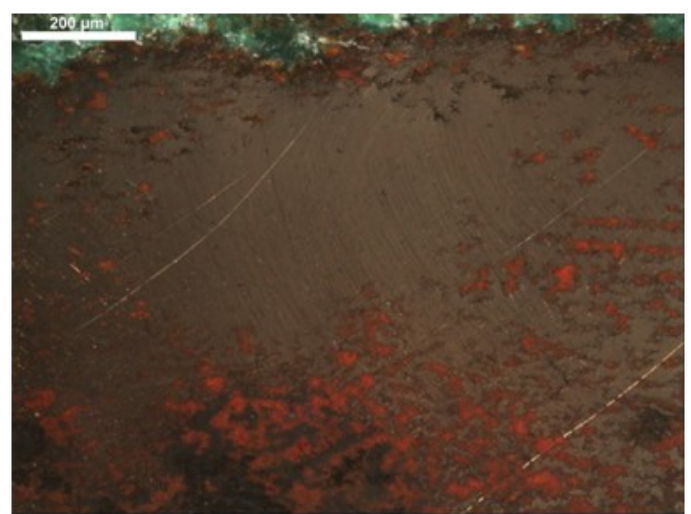

Fig. 9. Observação por microscopia ótica, sob luz polarizada, da extensão da camada de corrosão interna na haste 2013-0451 (100').

Fig. 9. Observation by optical microscopy, under polarized light, of the extension of internal corrosion in the rod 2013$0451\left(100^{\prime}\right)$.
Assim, e apesar de não ter sido ainda realizada a contrastação das zonas polidas, durante as observações em campo claro foi possível retirar algumas informações pertinentes quanto às técnicas de manufatura dos artefactos, para além da identificação de diferentes fases metálicas e inclusões.

Em todos os artefactos foi identificada a fase $\alpha$ do cobre, solução sólida de cobre com estanho, de tom rosa-amarelado. Em alguns artefactos de bronze foi também identificado o microconstituinte eutectóide $(\alpha+\delta)$. Comparativamente à fase $\alpha$, a fase $\delta$ apresenta um tom prateado (Fig. 10).

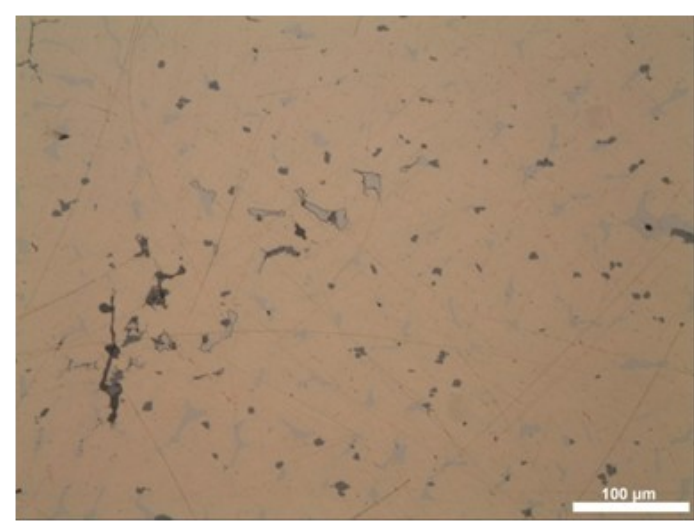

Fig. 10. Detalhe da microestrutura da argola 2013-0439 vista por microscopia ótica, sob campo claro, com a visualização de diferentes fases metálicas e presença de inclusões $\left(200^{\prime}\right)$. Fig. 10. Detail of the microstructure of the ring 2013-0439 observed by optical microscopy, under bright field, with visualization of different metallic phases and inclusions (200').

$\mathrm{Na}$ maioria dos objetos foram também detetadas inclusões de cor cinzenta escura, reconhecidos como sulfuretos de cobre (Fig. 10), inclusões típicas em artefactos deste período (FIGUEIREDO et al. 2011).

Em muitos dos artefactos, ainda sem uma contrastação artificial, a corrosão interna (inter e transgranular) revelou uma microestrutura constituída por grãos recristalizados de fase $\alpha$ sem evidências do microconstituinte eutectóide $(\alpha+\delta)$. A ausência de eutectóide para os teores de Sn registados é frequente em estruturas quimicamente mais homogéneas, sugerindo que a enformação da maioria dos objetos envolveu processamentos termomecânicos (forjagem + calor), conseguindo-se, assim, uma maior homogeneização química. Nalguns artefactos foi ainda possível observar bandas de deformação (Fig. 11), que podem dever-se à deformação provocada pelo uso do objeto ou demostrar que a última operação a que o material foi sujeito terá sido uma deformação (plástica) a frio. 


\section{CONCLUSÕES}

A aplicação da radiografia de raios $\mathrm{X}$, microEDXRF e microscopia ótica revelou-se adequada para obter informações pertinentes e complementares sobre o estado de preservação, composição e técnicas de manufatura dos artefactos metálicos de Moinhos de Golas.

A utilização da radiografia por raios $\mathrm{X}$ mostrou-se pertinente para o estudo das técnicas de manufatura de alguns artefactos e foi determinante na avaliação do estado de conservação de alguns objetos particulares na coleção, revelando corrosão localizada e fraturas que nem sempre são visíveis a olho nu, mas que poderão ter implicações no manuseamento e estado de conservação dos artefactos.

As análises por micro-EDXRF permitiram classificar o tipo de liga/metal em que cada artefacto foi fabricado, podendo constatar-se que a coleção é composta maioritariamente por bronzes (73\%), havendo alguns objetos em cobre (15\%) e outros em latão. De destacar a utilização de diferentes metais num mesmo artefacto (p. ex. no eventual freio) ou dentro de uma mesma tipologia de artefactos, como é o caso das argolas. A maioria dos artefactos em bronze possui teores de estanho entre $10 \%$ e $15 \%$, com baixos teores de impurezas, o que os relaciona com a metalurgia tradicional da Idade do Bronze Final do território português.

A presença de latões, aliada à presença de cobres e de bronzes, na mesma coleção, bem como a diversidade tipologica dos artefactos constitui uma particularidade que tando poderá relacionar-se com deposições em diferentes períodos cronológico -culturais, podendo alguns artefactos ser resultado de "depósitos" acidentais, como com a circulação de produtos exógenos a partir dos finais da Bronze Final, inícios da Idade do Ferro (entre os séculos VIII-VI a.C.) $)^{6}$. Tal irá constituir um ponto de partida para uma pesquisa mais aprofundada no desenvolvimento do estudo arqueometalúrgico em curso.

A observação da microestrutura dos artefactos através de microscopia ótica possibilitou um melhor entendimento do desenvolvimento da corrosão nos objetos e, embora ainda em fase de investigação, permitiu inferir alguns processos de manufatura, tendo sido possível apurar que a maioria dos artefactos sofreu ciclos de tratamento termomecânico.

De uma forma geral, o estado atual do estudo arqueometalúrgico permite constatar que a coleção de artefactos metálicos é bastante diversificada, sobretudo a nível material (tendo em conta os diferentes metais/ligas usados), possibilitando, sem dúvida, uma multiplicidade de questões, ainda por explorar.

\section{AGRADECIMENTOS}

Os autores agradecem à Fundação para a Ciência e a Tecnologia (FCT-MEC) as bolsas individuais SFRH/BPD/73245/2010, SFRH/ BPD/97360/2013 e SFRH/BD/65143/2009, concedidas a Elin Figueiredo e João Fonte, e o apoio financeiro concedido ao CENIMAT/I3N através do Projeto Estratégico LA25/2013-2014 (PEst-C/ CTM/LA0025/2013-2014). O trabalho foi ainda realizado no âmbito dos Projetos EARLYMETAL (PTDC/HIST-ARQ/110442/2008) e ENARDAS (PTDC/HISARQ/112983/2009), financiados pelo Programa Operacional Temático Fatores de Competitividade (COMPETE) e pelo Fundo Comunitário Europeu (FEDER).

\section{REFERÊNCIAS}

ADRIENS, A. 2005. Non-destructive analysis and testing of museum objects: an overview of 5 years of research. Spectrochimica Acta Part B, 60: 1503-1516.

Bettencourt, A.M.S. 1998. O conceito de Bronze Atlântico na Península Ibérica. In S. O. Jorge (ed.) Existe um Idade do Bronze Atlântico? Lisboa: IPA, 18-34.

Bottaini, C. 2012. Depósitos metálicos do Bronze Final (sécs. XIII-VIII a.C.) do Centro e Norte de Portugal. Aspectos sociais e arqueometalúrgicos. Coimbra: Faculdade de Letras da Universidade de Coimbra (Tese de doutoramento - policopiada).

Bronk, H.; RÖHRs, S.; BJEOUMIKHOV, A; LANGHOFF, N.; Schmalz, J.; Wedell, R.; Gorny, H.-E; Herold, A. \& WALDSCHLÄGER, U. 2001. ArtTAX - a new mobile spectrometer for energy-dispersive micro Xray fluorescence spectrometry on art and archaeological objects. Frenesius Journal of Analytical Chemistry, 371: 307-316.

CARdoso, J.L. 1995. O povoado do Bronze Final da Tapada da Ajuda, In I. Cordeiro et al. (eds.) A Idade do Bronze em Portugal. Discursos do Poder: Lisboa: SEC, IPM, 48.

Centre Technique des Industries des Alliages CuIVREUX. 1967. Atlas Metallographique des Alliages Cuivreux. Paris: Éditions Techniques des Industries de la Fonderie.

FANG, J. \& MCDONNELL, G. 2011. The colour of copper alloys. The Journal of the Historical Metallurgy Society, 45 Part 1: 52-61.

Figueiredo, E.; ARAúJo, M.F.; Silva, R.J.C. \& VilaÇA, R. 2013a. Characterisation of a Proto-historic bronze collection by micro-EDXRF. Nuclear Instruments and Methods in Physics Research B, 296: 26-31.

Figueiredo, E.; Silva, R.J.C.; AraúJo, M.F. \& FernanDES, F.M.B. 2013b. Multifocus Optical microscopy Applied to the Study of Archaeological Metals. Microscopy and Microanalysis, 19: 1248-1254.

${ }^{6}$ Vide, por exemplo, MONTERo-Ruiz \& PeREA (2007). 
Figueiredo, E.; Silva, R.J.C.; SenNa-Martinez, J.C. \& VAZ, J.L. 2011. Characterization of Late Bronze Age large size shield nails by EDXRF, microEDXRF and X-ray digital radiography. Applied Radiation and Isotopes, 69: 1205-1211.

Fonte, J.; Bettencourt, A.M.S. \& Figueiredo, E. 2013. Deposições Metálicas do Bronze Final no Vale do Assueira. O Caso do Sítio de Moinhos de Golas (Solveira, Montalegre, Norte de Portugal). Estudos do Quaternário, 9: 17-27.

LeChTMAN, H. 1996. Arsenic Bronze: Dirty copper or chosen alloy? A view from the Americas. Journal of Field Archaeology, 23: 477-514.

Lehmann, E. H.; Vontobel, P.; Deschler-Erb, E. \& SOARES, M. 2005. Non-invasive studies of objects from cultural heritage. Nuclear Instruments and Methods in Physics Research, A 542: 68-75.

Montero-Ruiz, I \& Perea, A. 2007. Brasses in the early metallurgy of the Iberian Peninsula. Metals and Mines: 136-139.

Piccardo, P.; Mille, B. \& Robbiola, L. 2007. Tin and copper oxides in corroded archaeological bronzes. Corrosion of metallic heritage artefacts. Cambridge: Woodhead Publishing Limited: 239-262.

Robbiola, L.; Blengino, J.M. \& Fiaud, C. 1998. Morphology and mechanisms of formation of natural patinas on archaeological Cu-Sn alloys. Corrosion Science, 12: $2083-2111$

Senna-Martinez. J.C. 1989. Pré-história Recente da bacia do médio e alto Mondego. Algumas contribuições para um modelo sociocultural. Lisboa: Faculdade de Letras da Universidade de Lisboa (tese de doutoramento - policopiada).

VALÉRIO, P.; ARAÚJO, M.F. \& CANHA, A. 2007. EDXRF and micro-EDXRF studies of Late Bronze Age metallurgical productions from Canedotes (Portugal). Nuclear Instruments and Methods in Physics Research, B 263: 477-482.

VILAÇA, R. 1995. Aspectos do povoamento da Beira Interior (Centro e Sul) nos Finais da Idade do Bronze. Trabalhos de Arqueologia 9. Lisboa: Instituto Português do Património Arquitectónico e Arqueológico.

WAYMAN, M.L. 2000. Archaeometallurgical contributions to a better understanding of the past. Materials Characterization, 45: 259-267. 